\title{
EXISTENCE OF FIXED POINTS OF NONEXPANSIVE MAPPINGS IN CERTAIN BANACH LATTICES
}

\author{
PAOLO M. SOARDI
}

\begin{abstract}
A fixed point theorem for nonexpansive mappings in dual Banach spaces is proved. Applications in certain Banach lattices are given.
\end{abstract}

1. Suppose $K$ is a subset of a Banach space $X$ and $T: K \rightarrow K$ is a nonexpansive mapping, i.e. $\|T(x)-T(y)\| \leqslant\|x-y\|, x, y \in K$. A wellknown theorem due to Kirk [1] states that, if $K$ is convex weakly compact (weak* compact when $X$ is a dual space) and has normal structure, then $T$ has a fixed point in $K$. In particular, if $X=L^{p}(1<p<\infty)$ Kirk's theorem applies to every bounded closed convex set $K$, while an analogous theorem was proved by Karlovitz [3, Corollary] in $l^{1}$. No result seems to be known in $L^{\infty}$.

In this paper we study the existence of fixed points of nonexpansive mappings in certain (complex) $\boldsymbol{A} M$-spaces. First we prove a general fixed point theorem for nonexpansive mappings in dual spaces and then we draw some consequences for nonexpansive mappings acting in (complex) $A M$ spaces which are dual to (complex) $A L$-spaces. These results imply, for instance, that every nonexpansive operator mapping into itself a closed ball $B \subseteq L^{\infty}$ has a fixed point in $B$, and every nonexpansive $T: L^{\infty} \rightarrow L^{\infty}$, which leaves invariant a weak* compact subset, has a fixed point (in $L^{\infty}$ ).

2. A real Banach lattice $X$ is called an $A M$-space (abstract- $m$-space) if $\|x \vee y\|=\|x\| \vee\|y\|$, for every $x, y \in X$ such that $x, y \geqslant 0$. Here and in the sequel $\vee$ and $\wedge$ denote the least upper bound and the greatest lower bound respectively. $X$ is said to be order complete if each set $A \subseteq X$ with an upper bound has a least upper bound. A complex $A M$-space is defined as the complexification of an $A M$-space.

Suppose $X$ is an order complete $A M$-space with unit (i.e. an element $e$ such that the unit ball at zero is the order interval $[-e, e]$ ); then $X$ is isometrically lattice isomorphic to the space $C_{R}(S)$ of all continuous real-valued functions defined on a compact Stonian space $S$.

For these and other facts about Banach lattices we refer to Schaefer's book [4].

Received by the editors March 19, 1978.

AMS (MOS) subject classifications (1970). Primary 47H10; Secondary 46B99, 46E05.

$K e y$ words and phrases. Fixed points, nonexpansive mappings, dual Banach spaces, $A M$-spaces, uniform relative normal structure. 
The following notation will be used throughout the paper: for a Banach space $X, B(x, r)$ denotes the closed ball centered at $x \in X$ of radius $r$; if $M \subseteq X$ is a nonvoid bounded subset, diam $M$ denotes the diameter of $M$ and co $M$ the closed convex hull of $M$.

Lemma. Suppose $X$ is the complexification of an order complete AM-space with unit. For every nonvoid bounded closed set $M \subseteq X$ there exists a point $z_{M} \in X$ with the following properties:

(a) $M \subseteq B\left(z_{M}, 2^{-1 / 2} \operatorname{diam} M\right)$.

(b) If, for some $y \in X, M \subseteq B\left(y, 2^{-1 / 2} \operatorname{diam} M\right)$, then $\left\|z_{M}-y\right\| \leqslant 2^{-1 / 2}$ $\operatorname{diam} M$.

A similar statement holds if $X$ is an order complete AM-space with unit, with $2^{-1 / 2}$ replaced by $1 / 2$.

Proof. We shall prove the lemma in the complex case, the real one being simpler. Let $C(S)$ denote the space of continuous complex-valued functions which represents $X$. Set:

$$
a_{1}=\bigvee_{f \in M} \operatorname{Re} f, \quad a_{2}=\bigwedge_{f \in M} \operatorname{Re} f, \quad a_{3}=\bigvee_{f \in M} \operatorname{Im} f, \quad a_{4}=\bigwedge_{f \in M} \operatorname{Im} f .
$$

By our assumption on $X$ the $a_{j}$ 's are continuous real-valued functions belonging to $C_{R}(S)$. Let $u=\left(a_{1}+a_{2}\right) / 2, v=\left(a_{3}+a_{4}\right) / 2$. We shall prove that $z_{M}=u+i v$ has the desired properties. Indeed, if $r=\operatorname{diam} M$, it is easy to see that $\left\|a_{1}-a_{2}\right\| \leqslant r$ and $\left\|a_{3}-a_{4}\right\| \leqslant r$ so that, for every $s \in S$ and $f \in M,|\operatorname{Re} f(s)-u(s)| \leqslant r / 2,|\operatorname{Im} f(s)-v(s)| \leqslant r / 2$; hence (a) holds.

Suppose now that $M \subseteq B\left(y, 2^{-1 / 2} \operatorname{diam} M\right)$ for some $y \in X$. Let $\varepsilon>0$ be arbitrarily small. For every $s \in S$ we can find a neighborhood of $s, V(s)$, such that $\left|a_{j}\left(t_{1}\right)-a_{j}\left(t_{2}\right)\right|<\varepsilon(j=1,2,3,4)$ and $\left|y\left(t_{1}\right)-y\left(t_{2}\right)\right|<\varepsilon$ whenever $t_{1}, t_{2} \in V(s)$.

Since $S$ is extremally disconnected, we have:

$$
a_{1}(s)=\inf _{U(s)} \sup _{t \in U(s)} \sup _{f \in M} \operatorname{Re} f(t)
$$

where $U(s)$ runs through a neighborhood base of $s$ (see [4, p. 107]). Hence there is a point $s_{1} \in V(s)$ and a function $f_{1, s} \in M$ such that: $\mid \operatorname{Re} f_{1, s}\left(s_{1}\right)-$ $a_{1}(s) \mid<\varepsilon$. Therefore

$$
\left|a_{1}(s)+i \operatorname{Im} f_{1, s}\left(s_{1}\right)-y(s)\right|<2 \varepsilon+2^{-1 / 2} r .
$$

Analogously, we can find points $s_{j} \in V(s)$ and functions $f_{j, s} \in M$ such that $\left|g_{j}(s)-y(s)\right|<2 \varepsilon+2^{-1 / 2} r$, where

$$
\begin{aligned}
& g_{j}(s)=a_{j}(s)+i \operatorname{Im} f_{j, s}\left(s_{j}\right) \quad \text { if } j=1,2, \\
& g_{j}(s)=\operatorname{Re} f_{j, s}\left(s_{j}\right)+i a_{j}(s) \quad \text { if } j=3,4 .
\end{aligned}
$$

Since the oscillation of the $a_{j}$ 's on $V(s)$ is less than $\varepsilon$, an elementary geometric argument shows that there is a number in the convex hull of the $g_{j}(s)$ 's whose distance from $z_{M}(s)$ is less than $\varepsilon$. Therefore $\left|z_{M}(s)-y(s)\right|<3 \varepsilon+2^{-1 / 2} r$ for all $s \in S$, whence (b). 
REMARK. It follows from the above construction that if $B$ is a closed ball containing $M$, then $z_{M} \in B$. Analogously, if $M$ is contained in some order interval $I=\{x \in X: a \leqslant x \leqslant b\}$, then $z_{M} \in I$ (in the case $X$ is an $A M$ space). Therefore we are led to the following definition.

Definition. A closed subset $K$ of a Banach space $X$ has uniform relative normal structure if there exists $c<1$ such that, for every nonvoid bounded closed subset $M \subseteq K$, there exists $z_{M} \in K$ with the following properties:

(a') $M \subseteq B\left(z_{M}, c \operatorname{diam} M\right)$.

(b') If, for some $y \in K, M \subseteq B(y, c \operatorname{diam} M)$, then $\left\|z_{M}-y\right\| \leqslant$ $c$ diam $M$.

This definition should be compared with the analogous definition in [2].

THEOREM. Suppose $X$ is a dual Banach space and $K \subseteq X$ a weak* closed set with uniform relative normal structure. Let $T: K \rightarrow K$ be a nonexpansive mapping which leaves invariant a weak* compact subset $M \subseteq K($ i.e. $T(M) \subseteq$ $M)$. Then there exists $u \in K$ such that $u=T(u)$.

Proof. Let $A_{0} \subseteq M$ be minimal among weak* compact invariant subsets of $M$. Then, if $\mathrm{cl}^{*}$ denotes the weak* ${ }^{*}$ closure,

$$
T\left(\mathrm{cl}^{*} T\left(A_{0}\right)\right) \subseteq T\left(A_{0}\right) \subseteq \mathrm{cl}^{*} T\left(A_{0}\right)
$$

so that $A_{0}=\mathrm{cl}^{*} T\left(A_{0}\right)$. Suppose $r$ is the diameter of $A_{0}$. Then the set $A=\left\{z \in K: A_{0} \subseteq B(z, c r)\right\}$ is nonvoid, since $z_{A_{0}} \in A$. Moreover $A$ is weak* compact, as an intersection of closed balls with $K$. Fix $\varepsilon>0$ arbitrarily. For every $z \in A$ and $x \in A_{0}$ there exists $y \in A_{0}$ such that:

$$
\|T(z)-x\|-\varepsilon \leqslant\|T(z)-T(y)\| \leqslant\|z-y\| \leqslant c r .
$$

Since $\varepsilon$ is arbitrary, $\|T(z)-x\| \leqslant c r$ and $T(A) \subseteq A$.

Define a set $H$ by $H=\{z \in A: A \subseteq B(z, c r)\}$. $H$ is nonvoid since $z_{A_{0}} \in H$. Let $A_{1}$ denote the intersection of all $w^{*}$ compact invariant subsets of $A$ containing $H$. An argument due essentially to Kirk [1] shows that diam $A_{1}$ $\leqslant c r$. Namely, let $F$ denote the set $\left\{z \in A_{1}: A_{1} \subseteq B(z, c r)\right\}$. $F$ contains $H$ and is weak ${ }^{*}$ compact (as an intersection of closed balls with $A_{1}$ ). Assume, by way of contradiction, that $T(z) \notin F$ for some $z \in F$; then the set $G=$ $B(T(z), c r) \cap A_{1}$ is weak* compact and contains $H$. Moreover, for every $x \in G:\|T(z)-T(x)\| \leqslant\|z-x\| \leqslant c r$, since $z \in F$. Hence $T(G) \subseteq G$ and, by the definition of $A_{1}, A_{1}=G$. But $T(z) \notin F$, so that $\|T(z)-x\|>c r$ for some $x \in A_{1}$, a contradiction. Consequently $T(F) \subseteq F$, and by the definition of $A_{1}$ again, $F=A_{1}$. Hence $\operatorname{diam} A_{1}=\operatorname{diam} F \leqslant c r$. Moreover $A_{0} \subseteq B(x, c r)$ for every $x \in A_{1}$. Repeating this construction, we define inductively a sequence of weak* compact subsets $A_{n} \subseteq K$ with the properties:

(i) diam $A_{n} \leqslant r c^{n}$,

(ii) $T\left(A_{n}\right) \subseteq A_{n}$,

(iii) $\|x-y\| \leqslant r c^{n}$, whenever $x \in A_{n}, y \in A_{n-1}$. 
If we pick a sequence of points $u_{n} \in A_{n}$ we have:

$$
\left\|u_{n}-u_{m}\right\| \leqslant r \sum_{n}^{m} c^{j} \quad(n<m) \text { and }\left\|u_{n}-T\left(u_{n}\right)\right\| \leqslant r c^{n} .
$$

Therefore $u_{n}$ converges in the norm topology to a point $u \in K$ such that $u=T(u)$.

We recall that an $A L$-space is a real Banach lattice such that $\|x+y\|=$ $\|x\|+\|y\|$ whenever $x, y \geqslant 0$. A complex $A L$-space is defined to be the complexification of a real $A L$-space. It is known [4] that the dual of a (complex) $A L$-space is a (complex) $A M$-space which satisfies the assumptions of the above lemma. Henceforth we have the following consequences.

Corollary 1. Suppose $X$ is the dual of a (complex) AL-space. Then, if $B \subseteq X$ is a closed ball and $T: B \rightarrow B$ is a nonexpansive mapping, $T$ has a fixed point in $B$.

Corollary 2. Suppose $X$ is the dual of an AL-space. If $I \subseteq X$ is a closed order interval and $T: I \rightarrow I$ is a nonexpansive mapping, $T$ has a fixed point in $I$.

Corollary 3. Suppose $X$ is the dual of a (complex) AL-space. If $T: X \rightarrow X$ is a nonexpansive mapping which leaves invariant a weak* compact subset of $X$, $T$ has a fixed point (in $X$ ).

RemarK. Suppose $(Y, \Sigma, \mu)$ is a $\sigma$-finite measure space; the dual of the (complex) $A L$-space $L^{1}(Y, \Sigma, \mu)$ is identified with $L^{\infty}(Y, \Sigma, \mu)$, so that the above corollaries hold with $L^{\infty}(Y, \Sigma, \mu)$ in place of $X$.

3. In this section we give an example of uniform relative normal structure in spaces which are not $A M$-spaces.

Suppose $X$ is a uniformly convex Banach space; denote by $X^{*}$ its dual space and by $\|\cdot\|$ and $\|\cdot\|^{*}$ the norms in $X$ and $X^{*}$ respectively. Let $Z$ denote the space of all sequences $z=\left(z_{1}, z_{2}, \ldots, z_{n}, \ldots\right), z_{n} \in X$, such that $\sup _{n}\left\|z_{n}\right\|=\|z\|_{\infty}<\infty$. $Z$ is not an $A M$-space unless $X$ itself is an $A M$-space. Moreover $Z$ is the dual of the space of all sequences $t=\left(t_{1}, t_{2}, \ldots, t_{n}, \ldots\right)$, $t_{n} \in X^{*}$, such that $\Sigma_{n}\left\|t_{n}\right\|^{*}<\infty$.

Proposition. $Z$ and every closed ball $B \subseteq Z$ have uniform relative normal structure.

Proof. The proof is achieved by generalizing the argument used in the lemma of $\S 2$. Suppose $C \subseteq Z$ is a closed nonvoid bounded set. Let $z=$ $\left(z_{1}, z_{2}, \ldots, z_{n}, \ldots\right)$ belong to $C$ and denote by $C_{n}$ the subset of $X$ described by $z_{n}$ as $z$ describes $C$. Since $X$ is uniformly convex, there exists $c<1$, independent from $C$ and $n$, such that there exist points $z_{C, n} \in \overline{\operatorname{co}} C_{n}$ with the property $\left\|z_{C, n}-z_{n}\right\| \leqslant c r$ for every $z_{n} \in C_{n}, n=0,1,2, \ldots$, (here we made $r=\operatorname{diam} C)$. Thus the point $z_{C}=\left(z_{C, 1}, \ldots, z_{C, n}, \ldots\right)$ has the property $\left(\mathrm{a}^{\prime}\right)$. On the other hand suppose that the point $y=\left(y_{1}, y_{2}, \ldots, y_{n}, \ldots\right)$ is such that $C \subseteq B(y, c r)$. It follows that $C_{n} \subseteq B\left(y_{n}, c r\right)$ for every $n$. By the 
definition of $z_{C, n}$, we have $z_{C, n} \in B\left(y_{n}, c r\right)$ too, so that $\left\|z_{C}-y\right\|_{\infty} \leqslant c r$. It is also clear that $z_{C} \in B$ if $C$ is contained in the closed ball $B$.

From this proposition it is possible to deduce the analogues of Corollaries 1 and 3.

\section{REFERENCES}

1. W. A. Kirk, A fixed point theorem for mappings which do not increase distance, Amer. Math. Monthly 76 (1965), 1004-1006.

2. Nonexpansive mappings and the weak closure of sequences of iterates, Duke Math. J. 36 (1969), 639-645.

3. L. A. Karlovitz, On nonexpansive mappings, Proc. Amer. Math. Soc. 59 (1976), 321-325.

4. H. H. Schaefer, Banach lattices and positive operators, Springer-Verlag, Berlin and New York, 1974.

Istituto Matematico dell'Università, via Saldini 50, 20133 Milano, Italy 